\title{
Epistemología de la Pedagogía Infantil
}

\author{
Fernando Arturo Romero Ospina ${ }^{1}$
}

\section{Resumen}

En este artículo se ha reflexionado sobre la Epistemología de Pedagogía Infantil para construir nuevos discursos y prácticas del saber pedagógico. Para lo cual fue preciso delimitar la pedagogía y educación; luego, se aclaró la cientificidad de la pedagogía, para poner en discusión la Epistemología de la Pedagogía Infantil , la importancia del discurso y prácticas que realiza el pedagogo infantil en su quehacer en la construcción de un saber, que permita erigir puentes con los otros saberes, como son los de psicología infantil, filosofía de la educación y didácticas. La metodología que se utiliza fue investigación documental que se encuentra en el método cualitativo a nivel interpretativo.

Palabras clave: pedagogía; infancia; educación; ciencia; aprendizaje; epistemología.

\section{Summary}

In this article, we have reflected on the epistemology of Children Pedagogy to construct new discourses and practices of pedagogical knowledge. For which it was necessary to delimit what is pedagogy and education; then, the scientific pedagogy was clarified in order to discuss the epistemology of Children Pedagogy, the importance of the discourse and practices carried out by the children's Pedagogue in his work, in the construction of a knowledge that allows the creation of bridges with other knowledges, such as those of children psychology, philosophy of education and didactics. The methodology used was based on a documentary research that is found in the qualitative method.

Keywords: Pedagogy; childhood; education; science; learning; epistemology.

\section{Introducción}

La Pedagogía Infantil tiene grandes aportes teóricos de la psicología cognitiva, psicología infantil, sociología, antropología educativa los cuales han permitido una reflexión de las prácticas pedagógicas que se desarrollan en la escuela y los procesos educativos.

1 Licenciado en Pedagogía Infantil de la Universidad Francisco José de Caldas, trabaja en la escuela Rural Cuaya en Suesca. E-mail: humanodelirio@yahoo.com 


\section{EDUCACIÓN}

También se destaca los postulados del grupo Historia de la Práctica Pedagógica en Colombia, quienes desarrollan grandes trabajos como los de Zuluaga y Echeverri (2003), sobre los discursos y prácticas pedagógicas; o los de Quiceno (2005), quien reflexiona pedagógicamente los conceptos de saber, asimismo y subjetividad desde la perspectiva de Foucault, pioneros en Colombia que han permitido nuevas posiciones sobre lo que es la pedagogía como saber y el maestro como investigador.

Sin embargo, el siguiente paso de la Pedagogía Infantil es construir una epistemología, donde existan discursos y prácticas propias permitiendo desarrollar objetos y metodologías de estudios. Esto es importante para que la Pedagogía Infantil deje de ser vista como un saber auxiliar de las psicologías y se permitan desde su reflexión en su quehacer abrir puentes de diálogo con los otros saberes como son las Psicología Infantil, Didácticas de las Ciencias y Filosofía de la Educación, donde la Pedagogía Infantil tenga una perspectiva investigadora de su práctica y discurso.

Esta aseveración también se justifica en la confusión que se da en los trabajos de grado de las licenciaturas de Pedagogía Infantil, en los aspectos didáctico y lo pedagógico, con lo cual "no se tiene claridad sobre si el maestro es un profesional de la pedagogía o un experto en didáctica” (Lucio, 1989, p.35).

Con este panorama se identifica problemáticas en el pedagogo infantil como: la falta de claridad y limitación en los términos que conforman la ciencia de la educación, la confusión terminológica que existe entre pedagogía y la educación, el aprendizaje y la didáctica que se evidencia en el quehacer del profesional en Pedagogía Infantil, instrumentalizado su saber y prácticas.

Desde esta perspectiva se podría caer en una didáctica sin pedagogía y en una pedagogía sin didáctica, estos dos escenarios son peligrosos como lo señala Lucio (1989) al decir:

Sin la perspectiva globalista e integradora de la visión pedagógica, la didáctica es un instrumento para enseñar mejor, sin preocuparse por el "a quién": el alumno es una maquina pensante, acumuladora (y productora, en el mejor de los casos) de conocimiento (1989, p.39).

En segundo lugar, se presenta "la subordinación de la pedagogía, por parte de las Ciencias de la Educación, se acentúa aún más en tanto saber es despojado de la posibilidad de pensar el eje maestro-escuela-sociedad-Estado-cultura" (Zuluaga, G. Olga. Echeverrri, S. A. Martínez B. A. et. al., 2003, p.26). El pedagogo infantil debe reflexionar sobre su saber, tejiendo y destejiendo su discurso desde sus prácticas que lo consolide como un investigador, para esto debe tener claridad sobre la epistemología de su quehacer. 
De esta forma el texto se divide en tres partes, la primera es definir qué es pedagogía, para esto buscará la claridad etimológica sobre los conceptos que acompañan las Ciencias de la Educación; la segunda es considerar la pedagogía como ciencia y la última es sobre la Epistemología de la Pedagogía Infantil y el reto del pedagogo infantil para consolidar su epistemología porque en su mirada del otro, encuentra sus discursos y prácticas, que debe ser traducido desde la pregunta constante sobre la pedagogía y la infancia.

\section{Revisión de literatura}

Se realizó una revisión literaria teniendo en cuenta tres factores: el concepto de pedagogía donde encontramos a la autora Olga Lucía Zuluaga (1999), quien plantea las prácticas pedagógicas, Ávila, R (1990) señala las diferencias entre: didáctica, educación y pedagogía, esta misma línea la seguirá Lucio R. (1989). En este mismo ítem son valioso los aportes de Jaeger, W (1957) y Platón (1986) sobre la importancia de la educación y en espacial del termino Virtud en el mundo griego.

El siguiente factor se relaciona con la ciencia con los aportes de clásicos como Foucault y sus estudios sobre la ciencias humanas, Popper, K. (1983) explica que es un teoría y la relevancia en las ciencias, Kuhn, T.S (1992) señalando la revoluciones científicas, autores que permiten una reflexión sobre la Pedagogía como Ciencia.

Finalmente la Pedagogía Infantil y sus retos epistemológicos, donde autores como Habermas, J. (1987) desde la teoría de la acción comunicativa permiten analizar el acto pedagógico como un acto comunicativo, Pérez, C (2004) indicara la construcción social de la infancia elemento importante en la Epistemología de la Pedagogía Infantil.

\section{Materiales y metodos}

Este texto está enmarcado en la investigación cualitativa, desde la investigación documental (ID), que se caracteriza por la revisión de documentos que tengan el tema o conceptos sobre el tema, "en este sentido trabaja con información cualitativa y es de naturaleza semiótica y hermenéutica" (Páramo,2011,p.196). Es un procedimiento de nivel interpretativo que intenta construir un diálogo entre los autores para generar un nuevo saber.

\section{Desarrollo temático}

Para establecer la Epistemología de Pedagogía Infantil se debe preguntar ¿qué es la pedagogía?, cuya definición ha causado una ambigüedad, con definiciones vagas e inestables; sin embargo, una de las que más se ha difundido como lo indica Planchard (1956) es que: "se define corrientemente la pedagogía como la ciencia y el arte de la 


\section{EDUCACIÓN}

educación" (p.20). No obstante, de esta definición clásica se debe diferenciar entre educación y pedagogía, posteriormente identificar si es una ciencia la pedagogía.

La diferencia que existe entre la pedagogía y la educación, no se trata de desligarlos en sus relaciones o encasillarlos; sin embargo, no es nada sencillo porque igual que el término pedagogía, la terminología de educación ha tenido diversas definiciones.

En la definición de educación encontramos por ejemplo lo de Durkheim al señalar que:

La educación es la acción ejercida por las generaciones adulta sobre las que todavía no están maduras para la vida social. Tiene por objeto suscitar y desarrollar en el niño cierto número de estados físicos, intelectuales y morales, que exigen de él la sociedad política en su conjunto y el medio especial, al que está particularmente destinado" (1976, p.70).

Se puede entonces vincular la educación con la transmisión de valores de una generación a otra generación, como lo señala Jaeger (1957) quien afirma que: "la educación es el principio mediante el cual la comunidad humana conserva y transmite su peculiaridad física y espiritual" (p.3). Esta definición tiene presente la perfeccionamiento del individuo, en la formación de un ser a partir de las características del ideal de una sociedad particular.

La noción de perfección es posible rastrearla en el mundo griego, Platón (1997) en el séptimo libro en La república plantea que sea el jefe y el rey se dará una educación más perfecta para el manejo de los demás para unir el estudio de la sabiduría al manejo de los negocios. Es decir que "la educación no es otra cosa que la forma aristotélica, progresivamente espiritualizada, de una nación.” (Jaeger, 1957, p.20).

Este ideal está enmarcado desde el concepto crucial del areté, que en castellano podría traducirse como virtud, un concepto no ligado a lo puramente moral, parafraseando a Jaeger (1957) sino al heroísmo, como lo encontramos en las epopeyas de Homero de la Ilíada y Odiseo, que es la lucha del héroe por encontrar su camino y del hombre contra los dioses, se podría decir que del paso de lo mítico a la racional.

La virtud será una preocupación tan importante que en Menón diálogo de Platón, Menón le pregunta a Sócrates:

¿Podrías decirme, Sócrates, si la virtud es cosa que se enseña, o si no se enseña sino se practica, o si ni se practica ni se aprende, sino que la tienen los hombres por naturaleza o de algún otro modo? (Platón, 1986, p.1). 
Pregunta que da cuenta de la búsqueda de la virtud en el mundo griego, además "en el concepto de la areté se concentra el ideal educador de este período, en su forma más pura" (Jaeger, 1957, p.21), concepto que no se le atribuye a cualquier persona del común sino que estaba destinada a la nobleza y al heroísmo.

Con lo anterior, se puede resumir en términos de Lucio que: "la educación es ante todo una práctica social, que responde a, o lleva implícita, una determinada visión del hombre (de lo que he llamado su "crecer"). (1989, p.35). Sin embargo esta intensiones de una sociedad particular pueden no ser acordes con el sujeto, con lo cual existirán contradicciones y tensiones que se extenderán sobre lo que se le impone al sujeto y lo que quiere ser el sujeto desde su perspectiva.

En estas tensiones surgen las preguntas ¿cómo educar? ¿A quién educar? ¿Para qué educar? que generan discursos y prácticas que se efectuaran en espacios y tiempos determinados, convirtiéndose en saberes que configuraran nuevas formas de pensar el ¿cómo educar?, entonces "hay pedagogía cuando se reflexiona sobre la educación, cuando el "saber educar" implícito, se convierte en un "saber sobre la educación" (sobre el "cómo", "por qué", "hacia dóndes)" (Lucio, 1989, p.36).

Esta definición está emparentada con la de Foucault al definir la Pedagogía: "como la transmisión de una verdad que tiene por función dotar a un sujeto cualquiera de actitudes, de capacidades, de saberes que antes no poseía y que deberá poseer al final de la relación pedagógica”. (1994, p.101), entonces la pedagogía tiene prácticas y discursos, siendo importante aclarar que "entre nuestra intelectualidad existe la tradición equívoca de considerar la educación como la ciencia y como la práctica que dan cuenta de la relación escuela-sociedad y de los diferentes aspectos de la enseñanza" (Zuluaga, 1999, p. 17).

Con la anterior "la pedagogía no es sólo un discurso acerca de la enseñanza, sino también una práctica cuyo campo de aplicación es el discurso" (Zuluaga, 1999, p.10). Estos discursos son los saberes y las ciencias que se convertirán en didácticas, de esta forma "la pedagogía responde científicamente a la pregunta ¿cómo educar? La didáctica lo hace con la pregunta ¿cómo enseñar?” (Lucio, 1989, p.38).

La pregunta ¿cómo educar? no es tan sólo el reconocimiento de unas prácticas sociales que se creen son aprendidas pero son inconscientes, ya que las compartimos dentro de la sociedad sin un análisis pero "la pedagogía en cambio es siempre y necesariamente una reflexión consciente sobre las prácticas, los procesos, las instituciones o lo sistemas educativos" (Ávila, 1990, p.54).

Es decir que se hace pedagogía cuando existe reflexión sobre las prácticas y discursos que realiza el pedagogo, por esto "la educación es a la pedagogía como la práctica a 
la teoría" (Ávila, 1990, p.57). Esto significa que aunque es importante su diferenciación la pedagogía y la educación son elementos inseparables del quehacer del pedagogo.

\section{Argumento de la discusión: ¿pedagogía como ciencia?}

$\mathrm{Al}$ establecer la diferencia entre educación y pedagogía, lo que sigue es establecer si la pedagogía es una ciencia y con esto reflexionar la Epistemología de la Pedagogía Infantil, discusión que significa también la consolidación de la pedagogía en las ciencias humanas con unas epistemologías y metodologías particulares en sus investigaciones.

En primer lugar el estudio sobre el hombre conlleva diversas reflexiones, para poder comprender al hombres desde sus realidades particulares y generales, puesto que "las ciencias sociales no son un fin en sí mismas, sino un medio de conocimiento $\mathrm{y}$, crecientemente de conocimiento para la toma de decisiones que orientan el desarrollo de nuestra sociedad" (Bonilla y Rodríguez, 2005,p.6o), es decir que no busca una verdad absoluta sino posibilidades de discutir al ser humano en diversas facetas, en este contexto el pedagógico.

Con lo anterior, el estudio del hombre encuentra preguntas que cambian al mismo ritmo del hombre según el tiempo, su historia y su contexto, es más "el hombre es una invención cuya fecha reciente muestra con toda facilidad la arqueología de nuestro pensamiento" (Foucault, 1984, p.375). De esta forma se explica cómo se origina la preocupación de las ciencias humanas por el hombre recientemente.

Con lo anterior, las ciencias humanas no pueden pretender dominar objetos de estudios, sino comprender realidades sociales porque existe "la amenaza que le ronda es cosificar, reducir a objeto todo, hasta el hombre mismo." (Mardonas, 1991, p. 30). Por esto las ciencias humanas al plantearse los objetos de estudio como lo señala Foucault (1984) debe reconocer que:

El objeto de las ciencias humanas no es, pues, el lenguaje (hablando sin embargo por ellos solos), es ese ser que, desde el interior del lenguaje por el que está rodeado, se representa, al hablar, el sentido de las palabras o de las proposiciones que enuncia y se da, por último, la representación del lenguaje mismo (p.343).

Es así que las ciencias humanas se han abierto paso, construyendo sus epistemologías, dejando de lado las posturas positivistas del siglo XIX, lo cual no significa que vivan en una relatividad científica, sino que han construido otros discursos y metodologías que permiten en sus estudios una seriedad, formulando postulados que son debatibles y refutables en búsqueda de la comprensión de la realidad social, histórica y cultural del ser humano. 
En el caso de la Pedagogía existen diversas posturas sobre su cientificidad y sobre su marco teórico en el campo de las Ciencias Sociales, como lo señala Moreno (2009) quién considera que la Pedagogía no es una ciencia sino que es un lenguaje sin contenido, una jerga vacía, señalando la falta de un método científico de la pedagogía y de nombrar nuevos nombres a cosas que ya existen ya que según él "las pseudo ciencias sí necesitan inventar palabras nuevas para así ocultar la falta de ideas" (Moreno, 2009, p. 76).

Otra postura es la de Ávila (1990) quien plantea que "la pedagogía no es una ciencia pura, porque no está destinada a comprender ni explicar la naturaleza de las prácticas educativas" (p.62). Por eso Ávila considera la pedagogía como "teoría practica cuya función es orientar la acción” (1990, p.62). Sin embargo la pedagogía vista de esta forma se instrumentaliza su saber.

Con estas diversas definiciones, es importante subrayar que en este texto siguiendo Zuluaga (1999), se puede hablar de pedagogía:

(...) Como el discurso que posibilita al maestro ser el soporte de un saber especifico, circunscrito a las prácticas de saber, y como el lugar en las prácticas de saber, y como el lugar teórico que conceptualiza tanto acerca de la enseñanza como las múltiples relaciones conceptuales y prácticas con las cuales entra en relación la enseñanza (p.14).

Es decir que construye saberes desde su reflexión y práctica generando teorías para darle validez a su quehacer, que deben ser verificables y refutables en términos de Popper, dándole el estatus de Ciencia a la Pedagogía; entonces, para entender la pedagogía como ciencia se debe definir qué es la ciencia.

Como indica Popper (1983) al tener la tradición griega de la racionalidad, permite una liberación, donde los científicos han tenido la osadía de crear mitos o conjeturas, o teorías que se encuentran en contraste con el mundo de la experiencia común, la ciencia estará acompañada de las teorías.

Para Popper (1983) se requiere establecer una diferencia entre ciencia y pseudociencia, dudando del método empírico, que parte de la inducción es decir de la observación y la experimentación, ya que para este autor: "toda observación está impregnada de teorías: no existe una información pura, desinteresada, libre de teorías". (Popper, 1985, p.168), en el caso de la pedagogía existirá una interpretación de la observación de las realidades que se da en los espacios pedagógicos, que pueden ser refutables y verificables.

En consecuencia "es posible resumir todo lo anterior diciendo que el criterio para establecer el estatus científico de una teoría es su refutabilidad o testabilidad" (Popper, 1983, p. 61). Para él refutabilidad se referirá a trazar una línea divisoria, entre 


\section{EDUCACIÓN}

enunciados de ciencias empíricas y otros enunciados de carácter religioso, metafísico o pseudo-ciencia, por lo tanto:

El criterio de refutabilidad es una solución a este problema de la demarcación, sostiene que para ser colocados en el rango de científicos, los enunciados o sistemas de enunciados deben ser susceptibles de entrar en conflicto con observaciones posibles o concebibles." (Popper, 1983, p. 63).

Sin embargo, a Popper se le discute el término de falsabilidad de las teorías científicas, a partir de los postulados de Kuhn plasmadas en su texto las revoluciones científicas, ya que Kuhn (1992) hablara de las revoluciones científicas que surgen cuando existe un cambio de paradigma, "Para ser aceptada como paradigma, una teoría debe parecer mejor que sus competidoras; pero no necesita explicar y, en efecto, nunca lo hace todos los hechos que se puedan confrontar con ella" (Kuhn, 1992, p.44). Estos paradigmas deben ser validados por una comunidad científica.

Con esta visión la pedagogía tiene validez como ciencia cuando describe, analiza, reflexiona y propone desde sus prácticas pedagógicas teorías, que generan cambios y se re-estructura a partir de su verificación y refutación, por esto la pedagogía es un saber práctico-teórico, componentes inseparables del quehacer pedagógico.

Siguiendo con esta idea, esto lo hace construyendo relaciones con los saberes y con el "otro" que se le llaman estudiante, pero él "otro" no se reduce a objeto de estudio sino sujeto, ya que "el sujeto ya no es un objeto, es un sujeto para sí. El hombre se representa como sujeto, necesita construirse como sujeto, como otro; esto es lo que se llama subjetividad" (Quiceno, 2005, p.77) y en la práctica pedagógica se construye saber desde la subjetividades, elemento que no puede ser olvidado en la pedagogía.

\section{Reflexiones sobre el tema}

En este escenario se ubica la Epistemología de Pedagogía Infantil , que es la reflexión de las prácticas y discurso existentes, entre la pedagogía e infancia, ejes que no son fijos y que se movilizan constantemente dependiendo de las circunstancias políticas y sociales en que se desarrollan.

Por consiguiente la Pedagogía Infantil tiene entre sus focos de estudio la infancia que es una construcción social y no es un estado natural que se determina a nivel biológico: "la infancia, por el contrario, es algo variable desde el punto de vista histórico, cultural y social” (Pérez, 2004,p.150), significa que la Pedagogía Infantil construye unos procesos que se cristalizan en prácticas hacia la infancia, que no se reducen por la edad o estado de madurez o inmadurez, sino que reflexiona sobre las infancias que existen en los diferentes contextos sociales como son las infancias indígenas, las infancias campesinas entre otras. 
También la Pedagogía Infantil tiene espacios de acción que no se reducen a la escuela, pero que es un espacio innegablemente privilegiado que promueve aprendizajes donde "el aprendizaje escolar se caracteriza como la asimilación significativa e intencionada de experiencias sistematizadas que se obtiene por la apropiación de las estructuras simbólicas del saber" (Pérez, 2006,p.1), un aprendizaje diferente al que existe en la vida cotidiana, ya que este aprendizaje escolar busca una trascendencia interpretativa y crítica de la realidad.

En este escenario el quehacer del pedagogo infantil se hará fundamental, puesto que construirá un discurso sobre lo que entiende por infancia-aprendizaje-enseñanza convirtiéndose en paradigmas, importante este punto ya que "es, mediante el efecto paradigma que la personas selecciona lo que se ajusta a sus propios paradigmas excluyendo, muchas veces, otras alternativas que podrían enriquecer la consolidación del pensamiento y de la acción" (Quiñones, 2009, p.2).

El peligro entonces es la falta de discusión sobre lo que se entiende por infancia y pedagogía, cayendo en una parálisis paradigmática es decir:

Es una especie de enceramiento en idea rígidas, una bien llamada ceguera intelectual o afectiva" que impide la utilización del pensamiento analógico y metafórico como alternativa de cambio (Quiñones, 2009, p.3), ya que el pedagogo infantil puede convertir su práctica en instrumentalización del saber, para que exista una epistemología en la Pedagogía Infantil es necesario que el pedagogo sea un investigador.

También otra manifestación de la parálisis paradigmática es asumir un modelo pedagógico como un dogma de fe sin cuestionar sus posibilidades en el quehacer pedagógico, por esto considerar un modelo pedagógico como respuesta produce un encasillamiento sobre lo que se hace en la escuela y la Epistemología de la Pedagogía Infantil no es estática se moviliza con la pregunta constante que se genera en la práctica pedagógica.

Desde esta visión el estudiante o la infancia no puede ser visto desde lo incompleto, sino desde las potencialidades ya que "el alumno es una persona libre que desea saber. Es su voluntad la que decide aquello que ejecutará y no la manipulación externa que induce determinado comportamientos" (Pérez, 2006, p.1). Entonces el estudiante se ve como un protagonista de su enseñanza-aprendizaje.

De la misma manera, maestro no es un observador pasivo ya que tiene un rol una responsabilidad social, que ha sido vista desde tres funciones: "apóstol, pedagogo y funcionario" (Gallego, 1995, p.61). El maestro es un agente civil que cumplirá con el sagrado mandato de la formación del hombre del mañana, pero que también debe ser vigilado y puesto a prueba constantemente por la sociedad. 


\section{EDUCACIÓN}

En esta situación, se debe recordar que el maestro debe ser un investigador de su realidad social y "los espacios educativos brindan la posibilidad de seleccionar fenómenos sociales que se pueden constituir en el objeto de estudio de la investigación con la gran ventaja de sus ocurrencia en contexto educativo" (Quiñones, 2009, p.8). Entonces, el pedagogo infantil también es foco de estudio desde su rol, sus posturas en el saber y su didáctica.

Con estos focos de estudios se relacionan constantemente construyéndose mutuamente desde la comunicación, en este sentido Habermas indica que: "el mundo sólo cobra objetividad por el hecho de ser reconocido y considerado como uno y el mismo mundo por una comunidad de sujetos capaces del lenguaje y de acción" (1987, p.30). El estudio del pedagogo infantil cobra sentido cuando existe una comunidad que se comunica.

Entonces, el pedagogo infantil debe construir discursos desde la argumentación para entablar comunicación ya que: "el tipo de habla en que los participantes tematizan las pretensiones de validez que se han vuelto dudosas y tratan de desempeñarlas o de recusarlas por medio de argumentos" (Habermas,1987,p.37). Desde el argumento el maestro debe generar su quehacer pedagógico, su objeto de estudio.

El argumento además constituye una práctica pedagógica donde los participantes puedan generar posturas, preguntas e investigaciones, de esta forma la teoría y la práctica tiene sentido porque está en constante cambio. Por lo tanto el pedagogo infantil en esta postura será un mediador, que actúa para generar espacio de aprendizaje, construyendo posibilidad de acciones comunicativas, es decir "el concepto de acción comunicativa se refiere a la interacción de a lo menos dos sujetos capaces de lenguaje y de acción que (ya sea con medios verbales o con medios extraverbales) entablan una relación interpersonal" (Habermas, 1987, p.124).

Siguiendo esta idea el quehacer del pedagogo infantil es una acción comunicativa también, porque debe generarse procesos de diálogo entre los saberes y la realidad ya que: "el maestro enfrenta sus conocimientos pedagógicos al discurso de las "teorías" o de las "ciencias" y el instrumento que utiliza para ellos es el método de enseñanza" (Zuluaga, 1999, p.10). Es así que el pedagogo infantil tiene una ardua labor para poder definir su campo de acción y buscar construir una epistemología que le de solidez a su saber, y construir puentes con saberes afines a su área, sin instrumentalizar su saber.

\section{Reflexiones finales}

Podemos concluir que la construcción de la Epistemología de la Pedagogía Infantil no significa una pretensión de delimitación, sino una posibilidad de reflexión del profesional de la Pedagogía Infantil sobre su quehacer, en la construcción de un discurso propio vinculante como otros saberes de las Ciencias Sociales. Es por lo tanto, un reto 
que el pedagogo infantil reflexione y ponga a niveles académicos nuevos postulados desde su praxis, siendo necesario la construcción de metodologías desde interpretación y la hermenéutica, que permiten comprender la realidad social del acto pedagógico en cada uno de los contextos.

Por esto es importante que las facultades de educación y carreras afines, se piensen desde el interior: la epistemología, filosofía y metodología de sus saberes, ya que se está cayendo en la instrumentalización de los saberes en el campo educativo y pedagógico, como es el caso de la utilización de términos como pedagogía, educación, didáctica en forma discriminada, sin una claridad reinando la confusión y perdiendo su significado esencial.

Finalmente, el pedagogo infantil debe construir nuevos paradigmas en la educación y la pedagogía desde la reflexión de su quehacer, comprendiendo las diversas relaciones que se establecen en el proceso del aprendizaje como es reconocer el contexto, comprender a los estudiantes en sus estilos de aprendizaje, construir propuestas curriculares integrales, es decir debe asumir una posición política que den cuenta de las preguntas por la sociedad y el ser humano en la actualidad.

\section{Lista de referencias}

Ávila, R. (1990). ¿Qué es la pedagogía? Bogotá: Editorial nueva américa.

Bonilla, E., y Rodríguez, P. (2005). Más allá del dilema de los métodos: la investigación en ciencias sociales. Bogotá: Editorial Norma.

Durkheim, E (1976). Educación y sociología. Bogotá: Editorial babel.

Foucault, M (1984). Las palabras y las cosas una arqueología y las cosas. México: Siglo XXI.

Foucault, M (1994). Hermenéutica del sujeto. Madrid: Ediciones de la piqueta

Gallego, A. (1995). Y la escuela se hizo necesaria: en busca del sentido actual de la escuela. Santafé de Bogotá: Magisterio.

Habermas, J (1987). Teoría de la acción comunicativa I racionalidad de la acción y racionalización social. Madrid: Taurus.

Jaeger, W (1957). Paideia: los ideales de la cultura griega. México, Fondo de Cultura Económica.

Kuhn, T.S. (1992). La estructura de las revoluciones científicas. México, Fondo de Cultura Económico. 


\section{EDUCACIÓN}

Lucio, R. (1989). Educación y pedagogía, enseñanza y didáctica: diferencias y relaciones. Tomado revista de la Universidad de la Salle. No. 17. P. 35-46.

Páramo, P. (compilador) (2011). La investigación en ciencias sociales: estrategias de investigación. Bogotá: Universidad Piloto de Colombia.

Pérez, C. (2004). La construcción social de la infancia apuntes desde la sociología. Tempora, 7; diciembre. pp 149-168.

Pérez, J. (2006). Una visión desde la didáctica de las ciencias fácticas interestructuración alumno-conocimiento y el docente como mediador. Didáctica de las ciencias fácticas, en línea, disponible en: http://www.korion.com.ar/archivos/modelo_inter_korion.pdf, recuperado: 9 de junio del 2016.

Planchard, E. (1956). La pedagogía contemporánea. Madrid: ediciones Rialp.

Platón (1986). Menón. Madrid: centro de estudios constitucionales.

Platón (1997). La república. Bogotá: panamericana.

Quiceno, H. (2005). De los usos de Foucault para la práctica pedagógica. Un saber por qué no funciona la escuela. En Zuluaga, O. et al. (2005). Foucault, la pedagogía y la educación: pensar de otro modo. Bogotá: Magisterio.

Quiñones, M. (2009). Parálisis paradigmática y su incidencia en el fluir de la creatividad en contexto educativo. Revista digital Educación y futuro, p.p 1-21.

Popper, K. (1985). La racionalidad de las revoluciones científicas. En Hancking, I. Revoluciones científicas. México: Fondo de cultura económico.

Popper, K. (1983). Conjeturas y refutaciones: el desarrollo del conocimiento científico. Buenos Aires: Paidós.

Mardonas, J.M. (1991). Filosofía de las ciencias humanas y sociales. Barcelona: Anthropos.

Moreno, R (2009). ¿Qué es la pedagogía? Tomado revista foro de educación., No. 11., pp. 67-83.

Zuluaga, O. (1999). Pedagogía e historia: la historicidad de la pedagogía, la enseñanza, un objeto de saber. Bogotá: siglo del hombre editores, Anthropos, Editorial Universidad de Antioquía.

Zuluaga, G. Olga. Echeverrri, S. A. Martínez B. A. et al., (2003). Pedagogía y epistemología. Bogotá: Magisterio. 